\title{
Characterization of symplectic forms induced by some tangent $G$-structures of higher order
}

\author{
P.M. Kouotchop Wamba ${ }^{1}$, G.F. WANkAP NonO ${ }^{2}$ \\ ${ }^{1}$ Department of Mathematics, Higher Teacher Training college \\ University of Yaoundé 1, PO.BOX 47 Yaoundé, Cameroon \\ ${ }^{2}$ Department of Mathematics and Computer Science, Faculty of Science \\ University of Ngaoundéré, PO.BOX 454 Ngaoundéré, Cameroon
}

wambapm@yahoo.fr, georgywan@yahoo.fr

Abstract: Let $(M, \omega)$ be a symplectic manifold induced by an integrable $G$-structure $P$ on $M$. In this paper, we characterize the symplectic manifolds induced by the tangent lifts of higher order $r \geq 1$ of $G$-structure $P$, from $M$ to $T^{r} M$.

Key words: Complete lifts of differential forms, prolongations of functions, vector fields and $G$ structures.

MSC (2020): 53C15; secondary 53C75, 53D05, 53D17.

\section{INTRODUCTION}

Let $M$ be a smooth manifold of dimension $n \geq 1$. The tangent bundle of order $r$ of $M$ is the $n(r+1)$-dimensional manifold $T^{r} M$ of $r$-jets at $0 \in \mathbb{R}$ of differential mapping $\varrho: \mathbb{R} \rightarrow M$. We denote by $\pi_{r, M}: T^{r} M \rightarrow M$ the canonical projection defined by $\pi_{r, M}\left(j_{0}^{r} \varrho\right)=\varrho(0)$. Let $\left(U, x^{i}\right)$ be a local coordinate system of $M$, we denote by $\left(x^{i}, x_{\alpha}^{i}\right)$ the adapted local coordinate of $T^{r} M$ over the open set $T^{r} U$, we have:

$$
\left\{\begin{aligned}
x^{i}\left(j_{0}^{r} \varrho\right) & =x^{i}(\varrho(0)), \\
x_{\beta}^{i}\left(j_{0}^{r} \varrho\right) & =\left.\frac{1}{\beta !} \frac{d^{\beta}}{d t^{\beta}}\left(x^{i} \circ \varrho\right)(t)\right|_{t=0} .
\end{aligned}\right.
$$

The differential geometry of the tangent bundles of higher order has been extensively studied by many authors, for instance I. Kolar ([1]), Morimoto ([3] and [4]). It plays an essential role in the description of a Lagrangian formalism of higher order. On the other hand, we denote by $F M$ the frame bundle of $M$, it is $\operatorname{GL}(n)$-principal bundle, where $\operatorname{GL}(n)$ is a linear Lie group of $\mathbb{R}^{n}$. Let $G$ be

ISSN: 0213-8743 (print), 2605-5686 online

(C) The author(s) - Released under a Creative Commons Attribution License (CC BY-NC 3.0) 
a Lie sub-group of linear group, we recall that, a $G$-structure on $M$ is a sub- $G$ principal bundle $\left(P, M, p_{M}\right)$ of $F M$. We know that some structures of classical differential geometry can be described by some $G$-structures. For instance, the almost complex structures, the Riemannian structures, the symplectic structures and regular foliations. That why, the study of tangent lifts of higher order of these structures has their importance in the calculus of variations and some problems connected as the Hamiltonization problems of higher order.

By the canonical linear action $\rho_{n}: \operatorname{GL}(n) \times \mathbb{R}^{n} \rightarrow \mathbb{R}^{n}$, we define an injective morphism of Lie groups $j_{n}^{\langle r\rangle}: T^{r}(\mathrm{GL}(n)) \rightarrow \mathrm{GL}(n(r+1))$ such that, for any manifold $M$, we have (see [3]) a natural principal bundle morphism $j_{M}^{\langle r\rangle}$ : $T^{r} F M \rightarrow F T^{r} M$ over $i d_{T^{r} M}$. The mapping is an embedding. Let $\left(P, M, p_{M}\right)$ be a $G$-structure, we set

$$
\begin{gathered}
G^{r}=j_{n}^{\langle r\rangle}\left(T^{r} G\right), \\
\mathcal{T}^{r} P=j_{M}^{\langle r\rangle}\left(T^{r} P\right) .
\end{gathered}
$$

The principal bundle $\left(\mathcal{T}^{r} P, T^{r} M, G^{r}\right)$ is a $G^{r}$-structure on $T^{r} M$. It is called tangent $G$-structure of order $r$. In [3], it has showed that the tangent $G$ - structure of order $r$ is integrable if and only if the initial structure is integrable. In particular, this prolongation generalize simultaneously the tangent lifts of higher order of almost complex structures, Riemannian structures, symplectic structures and regular foliations. In the particular case where $r=1$, A. Morimoto has shown the following result.

TheOREM 1. ([2]) Let $G$ be a Lie group generated by all elements $u \in$ $G L(n)$ invariant with respect to a bilinear form $f$ on $\mathbb{R}^{2 m}$. We denote by $\left(\mathcal{T} P, T M, \mathcal{T} p_{M}\right)$ the tangent lift of integrable $G$-structure $\left(P, M, p_{M}\right)$. Let $\omega_{G}$ be a symplectic form induced by $P$ and $\omega_{G^{1}}$ the symplectic form induced by $\left(\mathcal{T} P, T M, \mathcal{T} p_{M}\right)$. We have:

$$
\omega_{G^{1}}=\left(\omega_{G}\right)^{(c)}
$$

where $\left(\omega_{G}\right)^{(c)}$ is the complete lift of $\omega_{G}$ from $M$ to $T M$.

In the case where $r \geq 2$, we do not have similar result. In this paper, we propose a generalization of this result. Thus, this paper is structured as follows. In Section 2, we recall some prolongations of differential forms from a manifold $M$ to $T^{r} M$. In Section 3 , we prove the main result of this paper. 


\section{Complete lift of Differential FORMS}

In this section we recall briefly the main result of A. Morimoto [4, about lifts of functions, vector fields and differential forms to the tangent bundle of higher order. These results will be used in the main section. Let $M$ be a smooth manifold of dimension $m \geq 1$. Let $f$ be a function of class $C^{\infty}$ and $\alpha \in\{0, \ldots, r\}$, we define the $\alpha$-lift $f^{(\alpha)}$ as the function on $T^{r} M$ given by the formula

$$
f^{(\alpha)}\left(j_{0}^{r} \varrho\right)=\left.\frac{1}{\alpha !} \frac{d^{\alpha}}{d t^{\alpha}}(f \circ \varrho)(t)\right|_{t=0} .
$$

If $\alpha$ is negative, then we set $f^{(\alpha)}=0$.

The family of $\alpha$-lifts of functions is very important because, if $\bar{X}$ and $\bar{Y}$ are vector fields such that $\bar{X}\left(f^{(\alpha)}\right)=\bar{Y}\left(f^{(\alpha)}\right)$ for all functions $f$ on $M$ and $\alpha=0, \ldots, r$, then $\bar{X}=\bar{Y}$.

Proposition 1. Let $X \in \mathfrak{X}(M)$ and $0 \leq \alpha \leq r$. There is one and only one vector field $X^{(\alpha)}$ on $T^{r} M$ such that:

$$
X^{(\alpha)}\left(f^{(\beta)}\right)=(X f)^{(\beta-\alpha)}
$$

for any $f \in C^{\infty}(M)$ and $0 \leq \beta \leq r$.

\section{Proof. See [4].}

The vector field $X^{(\alpha)}$ is called $\alpha$-prolongation of $X$. For some properties of $X^{(\alpha)}$, see for instance [1] or 4]. Let $\left(U, x^{i}\right)$ be a local coordinate system of $M$ and $\left(x^{i}, x_{\beta}^{i}\right)$ be a local coordinate system on $T^{r} M$ over $T^{r} U$ induced by $\left(U, x^{i}\right)$ such that $X=a^{i} \frac{\partial}{\partial x^{i}}$, with $a^{i} \in C^{\infty}(U)$ we have:

$$
X^{(\alpha)}=\left(a^{i}\right)^{(\beta-\alpha)} \frac{\partial}{\partial x_{\beta}^{i}} .
$$

Proposition 2. Let $\omega$ be a differential form of degree $p$. It exists on $T^{r} M$ one and only one differential form of degree $p$ denoted by $\omega^{(c)}$ verifying:

$$
\omega^{(c)}\left(X_{1}^{\left(\beta_{1}\right)}, \ldots, X_{p}^{\left(\beta_{p}\right)}\right)=\left(\omega\left(X_{1}, \ldots, X_{p}\right)\right)^{\left(r-\left(\beta_{1}+\cdots+\beta_{p}\right)\right)}
$$

for all $X_{1}, \ldots, X_{p} \in \mathfrak{X}(M)$ and $\beta_{1}, \ldots, \beta_{p} \in\{0, \ldots, r\}$.

Proof. See [4]. 
Definition 1. The differential form $\omega^{(c)}$ is called complete lift of $\omega$ from $M$ to $T^{r} M$.

Let $\left\{x^{1}, \ldots, x^{n}\right\}$ be a local coordinates system of $M$ such that, the local expression of $\omega$ is given by:

$$
\omega=\omega_{i_{1} \cdots i_{p}} d x^{i_{1}} \wedge \cdots \wedge d x^{i_{p}}
$$

The local expression of $\omega^{(c)}$ is given by:

$$
\omega^{(c)}=\sum_{\beta_{1}+\cdots+\beta_{p}+\beta=r}\left(\omega_{i_{1} \cdots i_{p}}\right)^{(\beta)} d x_{\beta_{1}}^{i_{1}} \wedge \cdots \wedge d x_{\beta_{p}}^{i_{p}} .
$$

In the particular case where $p=2$, i.e., $\omega=\omega_{i j} d x^{i} \wedge d x^{j}$, then the differential form $\omega^{(c)}$ has the matrix form

$$
\left[\begin{array}{ccc}
\left(\omega_{i j}\right)^{(r)} & \cdots & \omega_{i j} \\
\vdots & . & \vdots \\
\omega_{i j} & \cdots & 0
\end{array}\right]
$$

It is called matrix representation of the complete lift $\omega^{(c)}$. In particular, if $\omega$ is non degenerate, then $\omega^{(c)}$ is also non degenerate.

Corollary 1. Let $\omega$ be a differential form of degree $p$ on $M$. We have:

$$
d\left(\omega^{(c)}\right)=(d \omega)^{(c)}
$$

\section{Proof. See [4].}

Remark 1. This corollary shows that, if $\omega$ is closed (resp. exact) then $\omega^{(c)}$ is closed (resp. exact). Thus, if $\omega$ is a symplectic form on $M$ then $\omega^{(c)}$ is a symplectic form on $T^{r} M$. On the other hand, the method employed for the description of the complete lifts of differential forms can be extended to a symmetric tensor. In particular for a symmetric tensor $g$ of type $(0,2)$ on $M$, if locally $g=g_{i j} d x_{i} \otimes d x_{j}$, then the complete lift $g^{(c)}$ is given by:

$$
g^{(c)}=\left(g_{i j}\right)^{(r-\alpha-\beta)} d x_{\alpha}^{i} \otimes d x_{\beta}^{j} .
$$




\section{The MAIN RESUlT}

Let $f$ be a bilinear symmetric (resp. skew symmetric $n=2 m$ ) non degenerate form on $\mathbb{R}^{n}$. In [3], the author shows that: if $G$ is a Lie sub-group in $\mathrm{I}(f)$, where $\mathrm{I}(f)$ is the Lie sub-group of all elements $u \in \mathrm{GL}(n)$ such that, for any $x, y \in \mathbb{R}^{n}$,

$$
f(u(x), u(y))=f(x, y),
$$

then $G^{r}$ is the Lie sub-group in the Lie group $\mathrm{I}\left(f^{\langle r\rangle}\right)$, where $\tau_{r}: T^{r}(\mathbb{R}) \rightarrow \mathbb{R}$ is defined by $\tau_{r}\left(j_{0}^{r} \varrho\right)=\left.\frac{1}{r !} \frac{d^{r}(\varrho)}{d t^{r}}(t)\right|_{t=0}$ and the bilinear form $f^{\langle r\rangle}=\tau_{r} \circ T^{r} f$ is symmetric (resp. skew symmetric).

Remark 2. Let $\left(P, M, p_{M}\right)$ be a $G$-structure, where $G \subset I(f)$ as above, $f$ is skew symmetric and non degenerate $(n=2 m)$. Let $\phi: M \rightarrow P$ be a section, for any $x \in M$ the map

$$
\begin{aligned}
T_{x} M \times T_{x} M & \longrightarrow \mathbb{R} \\
\left(u_{x}, v_{x}\right) & \longmapsto f\left(\phi(x)^{-1}\left(u_{x}\right), \phi(x)^{-1}\left(v_{x}\right)\right)
\end{aligned}
$$

is bilinear, skew symmetric and does not depend of $\phi$. We denote it by $\omega_{G}(x)$, in particular we obtain an almost symplectic form $\omega_{G}$ on the manifold $M$. If $\left(P, M, p_{M}\right)$ is integrable, then $\omega_{G}$ is a symplectic form on $M$. It is called symplectic form induced by $\left(P, M, p_{M}\right)$.

Theorem 2. Let $f$ and $f^{\langle r\rangle}$ be as above. Let $G$ be a Lie subgroup of the Lie group $I(f)$. Let $\left(P, M, p_{M}\right)$ be an integrable $G$-structure on $M(\operatorname{dim} M=$ $n=2 m)$. We denote by $\omega_{G}$ and $\omega_{G^{r}}$ the symplectic forms induced by $P$ and $\mathcal{T}^{r} P$ respectively. We have:

$$
\omega_{G^{r}}=\left(\omega_{G}\right)^{(c)} .
$$

Let $\phi \in \Gamma(P)$, where $\Gamma(P)$ denote the space of smooth sections of $P$, we have:

$$
\phi(x)\left(e_{j}\right)=\phi_{j}^{i}(x)\left(\frac{\partial}{\partial x^{i}}\right)_{x} .
$$

By the same process defined in [3], we prolong $\phi$ from $P$ to $\mathcal{T}^{r} P$ and we obtain the section $\Phi$ on $\mathcal{T}^{r} P$. The matrix form of $\Phi$ is given by:

$$
\left[\begin{array}{ccc}
\phi_{j}^{i} & \cdots & 0 \\
\vdots & \ddots & \vdots \\
\left(\phi_{j}^{i}\right)^{(r)} & \cdots & \phi_{j}^{i}
\end{array}\right] .
$$


Thus, we deduce that, for any $i, j \in\{1, \ldots, n\}$ and $\alpha, \beta \in\{0, \ldots, r\}$, we have:

$$
\Phi_{j+n \beta}^{i+n \alpha}=\left(\phi_{j}^{i}\right)^{(\alpha-\beta)} .
$$

Lemma. We denote by $\left(e_{\ell+n \alpha}\right)_{\ell, \alpha}$ the canonical basis of $\mathbb{R}^{n(r+1)}$. Using the matrix form of $\Phi$, we deduce that:

$$
\Phi\left(e_{j+n \alpha}\right)=\left(\phi_{j}^{i}\right)^{(\alpha-\beta)} \frac{\partial}{\partial x_{\beta}^{i}}
$$

for any $j \in\{1, \ldots, n\}$ and $\alpha \in\{0, \ldots, r\}$.

Proof. It comes from previous equation.

Proof of Theorem 2, We denote by $f: \mathbb{R}^{n} \times \mathbb{R}^{n} \rightarrow \mathbb{R}$ the bilinear skew symmetric, non degenerate form and $\left(a_{i j}\right)_{1 \leq i, j \leq n}$ its matrix form $(n=2 m)$. The matrix form of the bilinear skew symmetric form $f^{\langle r\rangle}: \mathbb{R}^{n(r+1)} \times \mathbb{R}^{n(r+1)} \rightarrow \mathbb{R}$ is given by:

$$
\left[\begin{array}{ccc}
0 & \cdots & a_{i j} \\
\vdots & . \cdot & \vdots \\
a_{i j} & \cdots & 0
\end{array}\right]
$$

We have, for any $i, j \in\{1, \ldots, n\}$ and $\alpha, \beta \in\{0, \ldots, r\}$,

$$
f^{\langle r\rangle}\left(e_{i+n \alpha}, e_{j+n \beta}\right)=\delta_{r-\beta}^{\alpha} a_{i j}
$$

where $\delta_{r-\beta}^{\alpha}$ is the Kronecker symbol. We denote by $\omega_{G^{r}}$ the symplectic form induced by an integrable $G^{r}$-structure $\mathcal{T}^{r} P$. For any $X \in T^{r} M$ and $\alpha, \beta \in\{0, \ldots, r\}$,

$$
\begin{aligned}
f^{\langle r\rangle}\left(e_{i+n \alpha}, e_{j+n \beta}\right) & = \\
& =f^{\langle r\rangle}\left(\Phi(X)^{-1}\left(\left(\phi_{i}^{k}\right)^{(\gamma-\alpha)} \frac{\partial}{\partial x_{\gamma}^{k}}\right), \Phi(X)^{-1}\left(\left(\phi_{j}^{s}\right)^{(\mu-\beta)} \frac{\partial}{\partial x_{\mu}^{s}}\right)\right) \\
& =\omega_{G^{r}}\left(\left(\phi_{i}^{k}\right)^{(\gamma-\alpha)} \frac{\partial}{\partial x_{\gamma}^{k}},\left(\phi_{j}^{s}\right)^{(\mu-\beta)} \frac{\partial}{\partial x_{\mu}^{s}}\right) \\
& =\left(\phi_{i}^{k}\right)^{(\gamma-\alpha)} \cdot\left(\phi_{j}^{s}\right)^{(\mu-\beta)} \omega_{G^{r}}\left(\frac{\partial}{\partial x_{\gamma}^{k}}, \frac{\partial}{\partial x_{\mu}^{s}}\right) .
\end{aligned}
$$


We deduce that:

$$
\left(\phi_{i}^{k}\right)^{(\gamma-\alpha)} \cdot\left(\phi_{j}^{s}\right)^{(\mu-\beta)} \omega_{G^{r}}\left(\frac{\partial}{\partial x_{\gamma}^{k}}, \frac{\partial}{\partial x_{\mu}^{s}}\right)=\delta_{r-\beta}^{\alpha} a_{i j} .
$$

We set

$$
\omega_{G^{r}}\left(\frac{\partial}{\partial x_{\gamma}^{k}}, \frac{\partial}{\partial x_{\mu}^{s}}\right)=\varpi_{k+n \gamma, s+n \mu} .
$$

In this case the equation 10 becomes:

$$
\left(\phi_{i}^{k}\right)^{(\gamma-\alpha)} \cdot\left(\phi_{j}^{s}\right)^{(\mu-\beta)} \varpi_{k+n \gamma, s+n \mu}=\delta_{r-\beta}^{\alpha} a_{i j} .
$$

Fact 1: For $\alpha=r$. If $\beta=r$, then the equation 12 becomes

$$
\phi_{i}^{k} \cdot \phi_{j}^{s} \varpi_{k+n r, s+n r}=0 .
$$

We deduce that

$$
\varpi_{k+n r, s+n r}=0 .
$$

Let $\beta \neq 0$, we suppose that, for any $\mu>\beta, \varpi_{k+n r, s+n \mu}=0$. We have:

$$
\begin{aligned}
\phi_{i}^{k} \cdot\left(\phi_{j}^{s}\right)^{(\mu-\beta)} \varpi_{k+n r, s+n \mu}= \\
\quad=\phi_{i}^{k} \cdot \phi_{j}^{s} \varpi_{k+n r, s+n \beta}+\sum_{\mu=\beta+1}^{r} \phi_{i}^{k} \cdot\left(\phi_{j}^{s}\right)^{(\mu-\beta)} \varpi_{k+n r, s+n \mu} \\
=\phi_{i}^{k} \cdot \phi_{j}^{s} \varpi_{k+n r, s+n \beta}=0 .
\end{aligned}
$$

We deduce that:

$$
\varpi_{k+n r, s+n \beta}=0 \quad \text { for any } \beta \neq 0 .
$$

For $\beta=0$, we have:

$$
\begin{aligned}
\sum_{\mu=0}^{r} \phi_{i}^{k} \cdot\left(\phi_{j}^{s}\right)^{(\gamma)} \varpi_{k+n r, s+n \gamma} & =\phi_{i}^{k} \cdot \phi_{j}^{s} \varpi_{k+n r, s}+\sum_{\mu=1}^{r} \phi_{i}^{k} \cdot\left(\phi_{j}^{s}\right)^{(\gamma)} \varpi_{k+n r, s+n \gamma} \\
& =\phi_{i}^{k} \cdot \phi_{j}^{s} \varpi_{k+n r, s}=a_{i j} .
\end{aligned}
$$

Now $\phi_{i}^{k} \cdot \phi_{j}^{s} \varpi_{k, s}=a_{i j}$, we deduce that:

$$
\varpi_{k+n r, s}=\varpi_{k, s} .
$$


Fact 2: If $\alpha=r-1$. By a similar calculus we obtain: for any $\beta \in$ $\{1, \ldots, r\}$, we have:

$$
\begin{cases}\varpi_{k+n(r-1), s+n \beta}=0 & \text { if } \beta \geq 2, \\ \varpi_{k+n(r-1), s+n \beta}=\omega_{k s} & \text { if } \beta=1 .\end{cases}
$$

For $\beta=0$, in the equation 12 , we have:

$$
\begin{aligned}
\left(\phi_{i}^{k}\right)^{(\gamma-\alpha)} \cdot\left(\phi_{j}^{s}\right)^{(\mu)} \varpi_{k+n \gamma, s+n \mu}= & \\
& =\left(\phi_{i}^{k}\right)^{(1)} \cdot\left(\phi_{j}^{s}\right)^{(\mu)} \varpi_{k+n r, s+n \mu}+\phi_{i}^{k} \cdot\left(\phi_{j}^{s}\right)^{(\mu)} \varpi_{k+n(r-1), s+n \mu} \\
& =\left(\phi_{i}^{k}\right)^{(1)} \cdot \phi_{j}^{s} \varpi_{k+n r, s}+\phi_{i}^{k} \cdot\left(\phi_{j}^{s}\right)^{(1)} \varpi_{k+n(r-1), s+n}+\phi_{i}^{k} \cdot \phi_{j}^{s} \varpi_{k+n(r-1), s} \\
& =\left(\phi_{i}^{k}\right)^{(1)} \cdot \phi_{j}^{s} \varpi_{k, s}+\phi_{i}^{k} \cdot\left(\phi_{j}^{s}\right)^{(1)} \varpi_{k, s}+\phi_{i}^{k} \cdot \phi_{j}^{s} \varpi_{k+n(r-1), s} \\
& =-\phi_{i}^{k} \cdot \phi_{j}^{s}\left(\varpi_{k, s}\right)^{(1)}+\phi_{i}^{k} \cdot \phi_{j}^{s} \varpi_{k+n(r-1), s}=0 .
\end{aligned}
$$

We deduce that:

$$
\varpi_{k+n(r-1), s}=\left(\varpi_{k, s}\right)^{(1)} .
$$

Fact 3: We conjecture that $\varpi_{k+n \alpha, s+n \beta}=\left(\varpi_{k, s}\right)^{(r-\alpha-\beta)}$. By induction, we fix $\alpha$ and we suppose that for any $\gamma>\alpha$ and $\lambda>\beta$, we have:

$$
\varpi_{k+n \gamma, s+n \lambda}=\left(\varpi_{k, s}\right)^{(r-\gamma-\lambda)} .
$$

Thus,

$$
\begin{aligned}
& \left(\phi_{i}^{k}\right)^{(\gamma-\alpha)} \cdot\left(\phi_{j}^{s}\right)^{(\mu-\beta)} \varpi_{k+n \gamma, s+n \mu} \\
& \quad=\phi_{i}^{k} \cdot \phi_{j}^{s} \varpi_{k+n \alpha, s+n \beta}+\sum_{\gamma, \lambda>\alpha, \beta}\left(\phi_{i}^{k}\right)^{(\gamma-\alpha)} \cdot\left(\phi_{j}^{s}\right)^{(\lambda-\beta)} \varpi_{k+n \gamma, s+n \lambda} \\
& \quad=\phi_{i}^{k} \cdot \phi_{j}^{s} \varpi_{k+n \alpha, s+n \beta}+\sum_{\gamma, \lambda>\alpha, \beta}\left(\phi_{i}^{k}\right)^{(\gamma-\alpha)} \cdot\left(\phi_{j}^{s}\right)^{(\lambda-\beta)}\left(\varpi_{k, s}\right)^{(r-\gamma-\lambda)} \\
& \quad=\phi_{i}^{k} \cdot \phi_{j}^{s} \varpi_{k+n \alpha, s+n \beta}+\left(\phi_{i}^{k} \cdot \phi_{j}^{s} \varpi_{k, s}\right)^{(r-\alpha-\beta)}-\phi_{i}^{k} \cdot \phi_{j}^{s}\left(\varpi_{k, s}\right)^{(r-\alpha-\beta)} \\
& =\phi_{i}^{k} \cdot \phi_{j}^{s} \varpi_{k+n \alpha, s+n \beta}+\delta_{r-\beta}^{\alpha} a_{i j}-\phi_{i}^{k} \cdot \phi_{j}^{s}\left(\varpi_{k, s}\right)^{(r-\alpha-\beta)} .
\end{aligned}
$$

Using the equation 10 , we deduce that:

$$
\phi_{i}^{k} \cdot \phi_{j}^{s} \varpi_{k+n \alpha, s+n \beta}=\phi_{i}^{k} \cdot \phi_{j}^{s}\left(\varpi_{k, s}\right)^{(r-\alpha-\beta)} .
$$


Therefore $\varpi_{k+n \alpha, s+n \beta}=\left(\varpi_{k, s}\right)^{(r-\alpha-\beta)}$. Thus, we deduce that

$$
\begin{aligned}
\omega_{G^{r}} & =\varpi_{i+n \alpha, j+n \beta} d x_{\alpha}^{i} \wedge d x_{\beta}^{j} \\
& =\left(\varpi_{k, s}\right)^{(r-\alpha-\beta)} d x_{\alpha}^{i} \wedge d x_{\beta}^{j} .
\end{aligned}
$$

Thus $\omega_{G^{r}}=\left(\omega_{G}\right)^{(c)}$.

Intrinsic second proof of Theorem 2. Let $\left(P, M, p_{M}\right)$ be an integrable $G$-structure as above. Because of the integrability of $P$ and the naturality, we may assume $M=\mathbb{R}^{n}$ and $P=\mathbb{R}^{n} \times G \subset \mathbb{R}^{n} \times G L(n)=L \mathbb{R}^{n}$. Then $T \mathbb{R}^{n}=\mathbb{R}^{n} \times \mathbb{R}^{n}$ and $\omega_{G}=\operatorname{id}_{\mathbb{R}^{n}} \times f: T \mathbb{R}^{n} \times \mathbb{R}^{n} T \mathbb{R}^{n}=\mathbb{R}^{n} \times\left(\mathbb{R}^{n} \times \mathbb{R}^{n}\right) \rightarrow \mathbb{R}$. More $T^{r} \mathbb{R}^{n}=\mathbb{R}^{n(r+1)}, \mathcal{T}^{r} P=T^{r} \mathbb{R}^{n} \times G^{r} \subset T^{r} \mathbb{R}^{n} \times G L\left(T^{r} \mathbb{R}^{n}\right)=L\left(T^{r} \mathbb{R}^{n}\right)$ and $T\left(T^{r} \mathbb{R}^{n}\right)=T^{r} \mathbb{R}^{n} \times T^{r} \mathbb{R}^{n}$ and $\omega_{G^{r}}=\operatorname{id}_{T^{r} \mathbb{R}^{n}} \times f^{\langle r\rangle}: T^{r} \mathbb{R}^{n} \times\left(T^{r} \mathbb{R}^{n} \times\right.$ $\left.T^{r} \mathbb{R}^{n}\right)=T\left(T^{r} \mathbb{R}^{n}\right) \times_{T^{r} \mathbb{R}^{n}} T\left(T^{r} \mathbb{R}^{n}\right) \rightarrow \mathbb{R}$, where $f^{\langle r\rangle} \doteqdot \tau_{r} \circ T^{r} f: T^{r} \mathbb{R}^{n} \times$ $T^{r} \mathbb{R}^{n} \rightarrow \mathbb{R}$ and $\tau_{r}: T^{r} \mathbb{R} \rightarrow \mathbb{R}$ is the respective functional. Further, $\left(\omega_{G}\right)^{(c)} \doteqdot$ $\tau_{r} \circ T^{r}\left(\omega_{G}\right)$ modulo the exchange isomorphism $T\left(T^{r} M\right)=T^{r}(T M)$ and the product preserving identification $T^{r}\left(T M \times_{M} T M\right)=T^{r}(T M) \times_{T^{r} M} T^{r}(T M)$. Then

$$
\begin{aligned}
\left(\omega_{G}\right)^{(c)} & =\tau_{r} \circ T^{r}\left(\operatorname{id}_{\mathbb{R}^{n}} \times f\right) \\
& =\operatorname{id}_{T^{r} \mathbb{R}^{n}} \times\left(\tau_{r} \circ T^{r} f\right)=\operatorname{id}_{T^{r} \mathbb{R}^{n}} \times f^{\langle r\rangle}=\omega_{G^{r}} .
\end{aligned}
$$

The proof is complete.

Corollary. Let $G$ be a Lie group generated by all elements of linear group invariant with respect to some bilinear symmetric non degenerate form f. Let $\left(P, M, p_{M}\right)$ be a $G$-structure on $M$. We denote by $g_{G}$ and $g_{G^{r}}$ the pseudo Riemannian metric on $M$ and $T^{r} M$ induced by $P$ and $\mathcal{T}^{r} P$ respectively. We have:

$$
g_{G^{r}}=\left(g_{G}\right)^{(c)} .
$$

Proof. The proof is similar to the proof of Theorem 2 .

\section{ACKNOWLEDGEMENTS}

The authors would like to thank the anonymous reviewers for their valuable suggestions and remarks which improved the quality of this paper. 


\section{REFERENCES}

[1] I. Kolar, P. Michor, J. SlovaK, "Natural operations in differential geometry", Springer-Verlag, Berlin 1993.

[2] A. Morimoto, Prolongations of $G$-structures to tangent bundles, Nagoya Math. J. 32 (1968), 67-108.

[3] A. Morimoto, Prolongations of $G$-structures to tangent bundles of higher order, Nagoya Math. J. 38 (1970), 153-179.

[4] A. Morimoto, Liftings of some types of tensors fields and connections to tangent bundles of $p^{r}$-velocities, Nagoya Math. J. 40 (1970), 13 -31. 